\title{
IMAGING IN EPILEPSY
}

T M Salmenpera, J S Duncan

J Neurol Neurosurg Psychiatry 2005;76(Suppl III):iii2-iii10. doi: 10.1136/jnnp.2005.075135

 he epilepsies are common serious diseases of the brain, with an age adjusted prevalence of 4-8/1000 and an annual incidence of 20-50/100 000 in developed countries. Modern neuroimaging is central to the assessment of patients with epilepsy and has dramatically modified their management. Magnetic resonance imaging (MRI) can identify substrates underlying epilepsy, and guide clinicians in the determination of treatment and prognosis. The use of $x$ ray computed tomography (CT) has been diminished by the superior sensitivity and specificity of MRI. The results of all imaging studies should be correlated with clinical and neurophysiological data.

Epilepsies and epileptic syndromes are classified into focal and generalised. Seizures of focal origin begin in a specific cerebral area or network, most commonly in the temporal lobes. Focal epilepsies comprise $40-60 \%$ of all newly diagnosed cases. Up to $30 \%$ of these patients develop intractable epilepsy despite antiepileptic drug treatment. In patients with chronic intractable temporal lobe epilepsy (TLE) surgical treatment with removal of the epileptogenic lesion is vastly superior to medical treatment. Results in extratemporal epilepsy have been less favourable, particularly if there is not a discrete underlying structural abnormality. Therefore, visualisation of lesions that give rise to focal epilepsy and identification of patients who are suitable for surgical treatment are important goals in the imaging of epilepsy.

\section{$X$ RAY COMPUTED TOMOGRAPHY}

In the modern imaging of epilepsy CT is supplementary. CT is useful in acute situations when the suspected underlying cause of seizures is a neurological insult such as intracerebral haemorrhage, or abscess, and MRI is not readily available or cannot be acquired. Focal cortical calcifications can be identified with $\mathrm{CT}$, and scanning may provide complementary information for the diagnosis of tuberous sclerosis and Sturge-Weber syndrome. CT is not as sensitive or specific as MRI in identifying common epileptogenic abnormalities, such as small tumours, vascular malformations, malformations of cortical development (MCD), and lesions in the medial temporal lobe. Thus, CT should not be a first line investigation for epilepsy, when MRI is the modality of choice, unless there are contraindications to MRI, such as a cardiac pacemaker or cochlear implants.

\section{MAGNETIC RESONANCE IMAGING Indications}

In general, all patients who develop epilepsy or whose chronic epilepsy has not been fully assessed should be investigated with MRI. In patients with newly diagnosed epilepsy, MRI may identify an epileptogenic lesion in $12-14 \%$, but up to $80 \%$ of the patients with recurrent seizures have structural abnormalities evident on MRI. MRI is not required in patients with a definite electroclinical diagnosis of idiopathic generalised epilepsy, or benign childhood epilepsy with centrotemporal spikes, who go into early remission. Table 1 summarises the situations in which obtaining MRI is particularly important. If access to MRI is limited, essential indications for scanning are evidence of progressive neurological deficit and intractable focal seizures.

See end of article for authors' affiliations

Correspondence to: Professor John S Duncan, Department of Clinical and Experimental Epilepsy, Institute of Neurology, Queen Square, University College London, London WCIN 3BG, UK; j.duncan@ion.ucl.ac.uk

\section{Patients with intractable epilepsy evaluated for surgery}

All patients with intractable epilepsy considered for surgery should undergo high resolution structural MRI as success of the operative treatment is directly related to the ability to localise precisely the region of seizure onset and underlying structural abnormalities. Since a structural abnormality does not necessarily indicate the site of seizure origin, clinical, electroencephalogram (EEG), and other data need to be correlated with imaging. MR based functional imaging methods, positron emission tomography (PET) and single photon emission computed tomography (SPECT) can provide additional information and assist in generating hypotheses for invasive EEG if structural imaging is normal or equivocal, shows diffuse abnormalities, or if there is discordance between different modalities. Postoperative MRI is useful to identify the 
Table 1 Indications for magnetic resonance imaging (MRI) in patients with epilepsy. Source: International League Against Epilepsy, 1997

1 Focal onset of seizures

2 Onset of generalised or unclassified seizures in the first year of life, or in adulthood

Focal deficit on neurological or neuropsychological examination Difficulty in obtaining seizure control with first line antiepileptic drugs

5 Loss of seizure control or change in the seizure pattern

extent of a cortical resection or the presence of residual pathology, particularly if seizures continue after surgery.

\section{Techniques}

The sensitivity of MRI for detecting abnormalities depends on the pathological substrate, the MRI techniques applied, and the experience of the interpreting physician. A routine optimal MRI protocol should include T1 and T2 weighted, proton density and fluid attenuated inversion recovery (FLAIR) sequences. These contrasts need to be acquired in at least two orthogonal planes covering the whole brain, using the minimum slice thickness possible. An oblique coronal plane, orientated perpendicular to the long axis of the hippocampus, gives the best definition of medial temporal lobe structures. In general, Tl weighted images give the best definition of the anatomy and differentiate grey and white matter, while T2 weighted images provide high sensitivity for detecting pathology in the brain. A three dimensional $\mathrm{Tl}$ weighted volume sequence with a partition size of $1.5 \mathrm{~mm}$ or less should be included as these images may be reformatted in any orientation and used for post-acquisition processing such as measuring hippocampal volumes. FLAIR imaging produces heavy T2 weighting and suppresses signal from cerebrospinal fluid (CSF). This provides high lesion contrast in areas close to CSF and enables anatomical detail to be seen with greater conspicuity than with conventional T2 weighted sequences. Gadolinium does not improve the sensitivity of MRI in patients with epilepsy, but may be useful to characterise intracerebral lesions associated with breakdown of the blood-brain barrier.

Visual assessment of all images should be carried out in the knowledge of the clinical situation by a neuroimaging specialist who has expertise in the imaging of epilepsy. A systematic approach to image reporting is essential, in addition to identification of obvious lesions, as dual pathology is not uncommon. It is important to search for subtle cortical abnormalities, including focal atrophic abnormalities and dysplastic lesions without mass effect, and to evaluate the hippocampi regardless of other MR findings. Thick image slices, rotated orientation, and the presence of normal anatomical variation make visual assessment more difficult and increase the risk of misdiagnosis.

\section{Epileptogenic substrates}

In developed countries hippocampal sclerosis (HS) and malformation of cortical development (MCD) are the most common identified structural causes of intractable seizures, followed by vascular malformations, tumours and posttraumatic, inflammatory or ischaemic lesions.

\section{Hippocampal sclerosis}

Hippocampal sclerosis is characterised by neuronal cell loss and gliosis in CAl, CA3, and dentate hilus subfields of the hippocampus, and can be reliably identified with MRI. The hippocampus is best visualised by acquiring thin slices (1-3 mm) orthogonal to its long axis. The primary MRI features of HS are hippocampal atrophy, demonstrated with coronal Tl weighted images, and increased signal intensity within the hippocampus on T2 weighted images (fig 1). Additionally, decreased $\mathrm{Tl}$ weighted signal intensity and disruption of the internal structure of the hippocampus may be present. Other MRI abnormalities associated with hippocampal damage include atrophy of temporal lobe white matter and cortex, dilatation of the temporal horn, and a blurring of the grey-white border in the temporal neocortex. Atrophy of the amygdala and entorhinal cortex variably accompany hippocampal damage but may also occur in patients with TLE and normal hippocampi. FLAIR images provide an increased contrast between grey and white matter, and facilitate differentiation of the amygdala from the hippocampus.

\section{Quantitative analysis of HS}

Visual assessment may reliably detect hippocampal volume asymmetry of more than 20\%; however, lesser degrees of asymmetry require quantitative volumetric analysis. The use of contiguous thin slices increases the reliability of measurements and permits localisation of atrophy along the length of the hippocampus. It is necessary to correct hippocampal volumes for intracranial volume to identify symmetrical bilateral atrophy. Hippocampal volume reduction correlates with the severity of neuronal cell loss. Patients with unilateral hippocampal volume loss, no other imaging abnormality, and concordant clinical and EEG data have more than $70 \%$ chance of an excellent surgical outcome.

Quantification of T2 relaxation time is another objective way to assess hippocampal damage. The advantage of this technique is that hippocampal T2 (HT2) times are absolute values, which can be compared against control values. Increased HT2 reflects underlying gliosis and neuronal loss. HT2 prolongation also correlates with hippocampal volume loss. Both volumetry and $\mathrm{T} 2$ relaxometry techniques can be applied for identifying subtle amygdala pathology. Volumetric studies of the entorhinal cortex may identify occult damage ipsilateral to the seizure focus that is not evident on visual inspection.

\section{Malformations of cortical development}

There are a wide range of focal and generalised malformations. In focal cortical dysplasia (FCD) the key MRI findings are focal cortical thickening, simplified gyration, blurring of the cortical-white matter junction, and T2 prolongation in the underlying white matter, that often forms a cone tapering towards the lateral ventricle. Focal cortical dysplasia commonly involves the frontal lobe and, on occasion, co-exists with hippocampal sclerosis, giving rise to dual pathology. Complete surgical removal of FCD is accompanied by a high remission rate, but may be technically difficult and the epileptogenic zone may be more extensive than the abnormal area visible on MRI.

Several gene mutations have been identified underlying lissencephalies resulting in characteristic MRI patterns of agyria/pachygyria. In heterotopias, collections of normal neurons are situated in abnormal locations: subependymally (fig 2), subcortically, or diffusively (band heterotopia). Band heterotopia is an example of a generalised MCD that may be present in patients with mild epilepsy and normal intellect. 

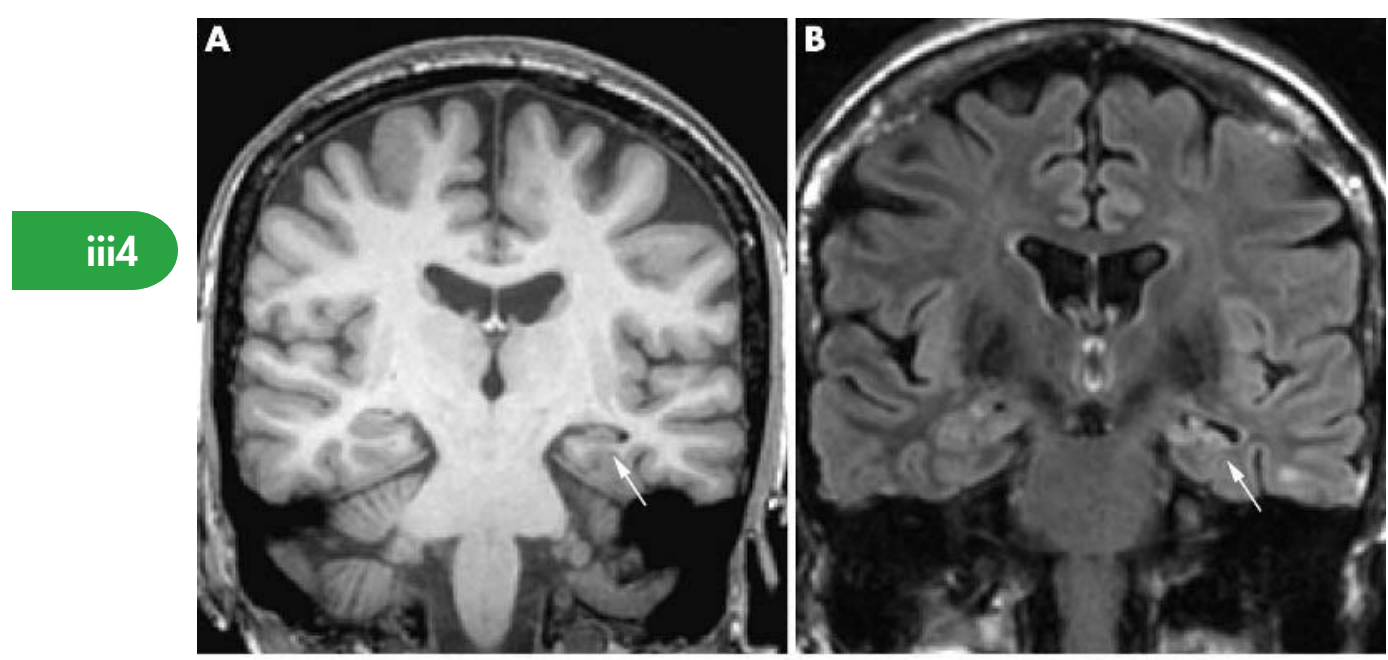

Figure 1 Left hippocampal sclerosis (3.0 T MRI). (A) Inversion recovery prepared (IRprep) $\mathrm{T} 1$ weighted acquisition showing an atrophic hippocampus (on right of image: arrow). (B) T2 weighted fluid attenuated inversion recovery (FLAIR) image demonstrating increased T2 weighted signal within the sclerotic hippocampus. (C) Early echo image from dual echo data (TE $30 \mathrm{~ms}$ ). (D) Later echo image from the same dual echo data (TE $120 \mathrm{~ms})$. Hippocampal T2 relaxation times may be obtained using the data from the dual echo sequence.
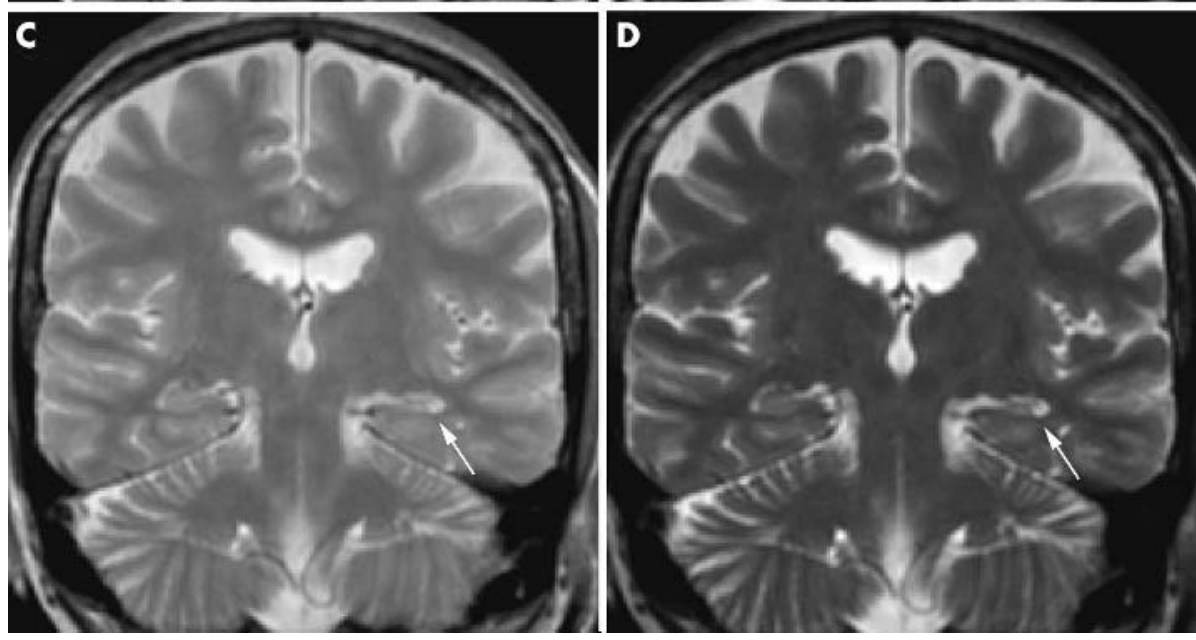

The abnormalities may be subtle and only be apparent if optimal MRI techniques are used.

Polymicrogyria with excessive number of small and prominent convolutions is a frequently identified MCD that develops secondary to abnormal late migration. Several syndromes of region specific symmetric polymicrogyria have

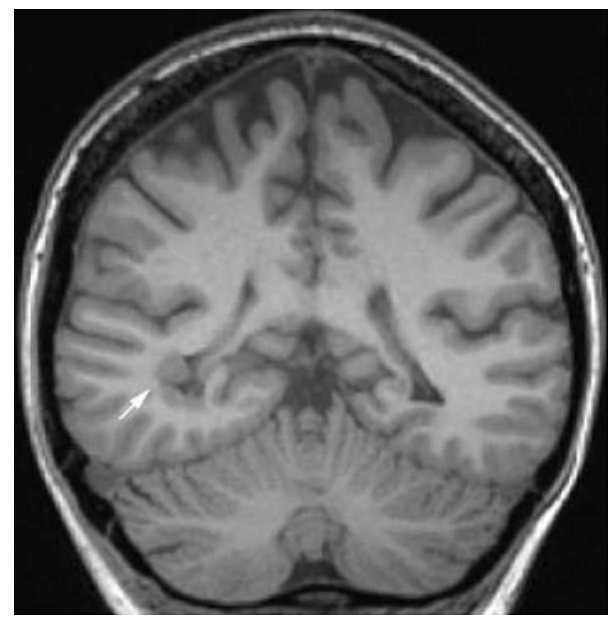

Figure 2 Subependymal heterotopia on the right (arrow) in coronal IRprep T1 weighted image (3.0 T MRI). Nodules isointense to grey matter are shown in the wall of the lateral ventricle. been reported, and some have been linked to specific genetic loci. Schizencephaly (cleft brain) is often found in MRI in conjunction with polymicrogyria.

\section{Primary brain tumours}

Patients with low grade primary brain tumours frequently have focal seizures as a presenting symptom. Underlying histopathologies include dysembryoplastic neuroepithelial tumours, ganglioglioma, gangliocytoma, and pilocytic and fibrillary astrocytoma. Most lesions have low signal on Tl and high signal on $\mathrm{T} 2$ weighted images, and are not usually associated with vasogenic oedema. Complete resection of the neoplasm and overlying cortex results in successful control of seizures in most cases. Dysembryoplastic neuroepithelial tumours are benign developmental tumours with features of a focal, circumscribed cortical mass that may indent the overlying skull (fig 3). Cyst formation and enhancement with gadolinium may occur. Calcification is present in some cases and may be more readily demonstrated with CT. Confident differentiation from low grade astrocytomas and gangliogliomas is not possible by MRI.

\section{Vascular malformations}

Cavernous haemangiomas (cavernomas) carry a good surgical prognosis, with $70 \%$ of the patients being seizure-free after removal of the lesion. Cavernous haemangiomas are circumscribed and have the characteristic appearance of a range of blood products on MRI (fig 4). The central part 

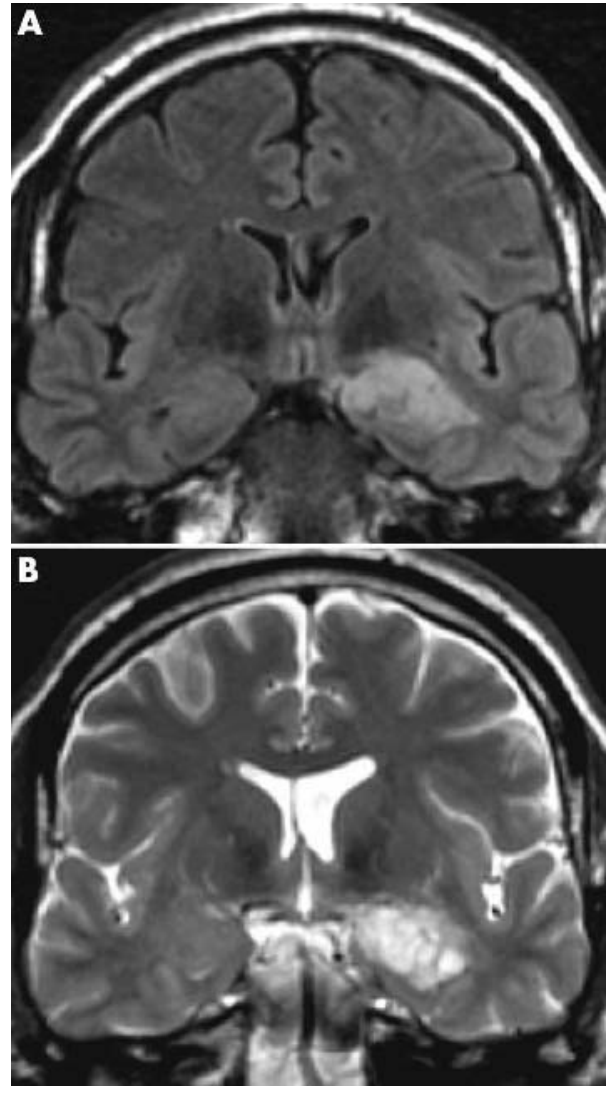

Figure 3 Dysembryoplastic neuroepithelial tumour on the left (coronal 3.0 T MRI). (A) T2 weighted FLAIR image. (B) Dual echo late echo image (TE 120). The images show a circumscribed multicystic mass with hyperintense signal in the region of the left amygdala.

contains areas of high signal on $\mathrm{T} 1$ and $\mathrm{T} 2$ weighted images, reflecting oxidised haemoglobin, with darker areas on $\mathrm{Tl}$ weighted images caused by deoxyhaemoglobin. The ring of surrounding haemosiderin appears dark on a T2 weighted image. Up to 50\% of cavernous malformations are multiple, and may occur on a familial basis. Arteriovenous malformations with high blood flow have a different and distinctive appearance with a nidus, feeding arteries, and draining veins.

\section{Acquired damage}

Focal or diffuse cortical damage can develop as a consequence of trauma, infarction, or infection of the central nervous system. Cerebrovascular disease associated with epilepsy is particularly common in older age groups. Worldwide, neurocysticercosis (fig 5) and tuberculomas are the most common causes of intractable focal epilepsy. These lesions have typical appearances on MRI that evolve with time and which, unless calcified, may resolve. MRI has a prognostic value in identifying and characterising the lesions.

\section{Serial MRI studies of structural brain changes in epilepsy}

Longitudinal quantitative MRI studies provide a tool for the study of structural changes over time to determine the effects of epilepsy on the brain. A longstanding unanswered question in epilepsy research is whether focal or secondarily generalised seizures cause irreversible neuronal injury. Previous cross sectional MRI studies have inferred that more severe hippocampal damage is associated with a longer
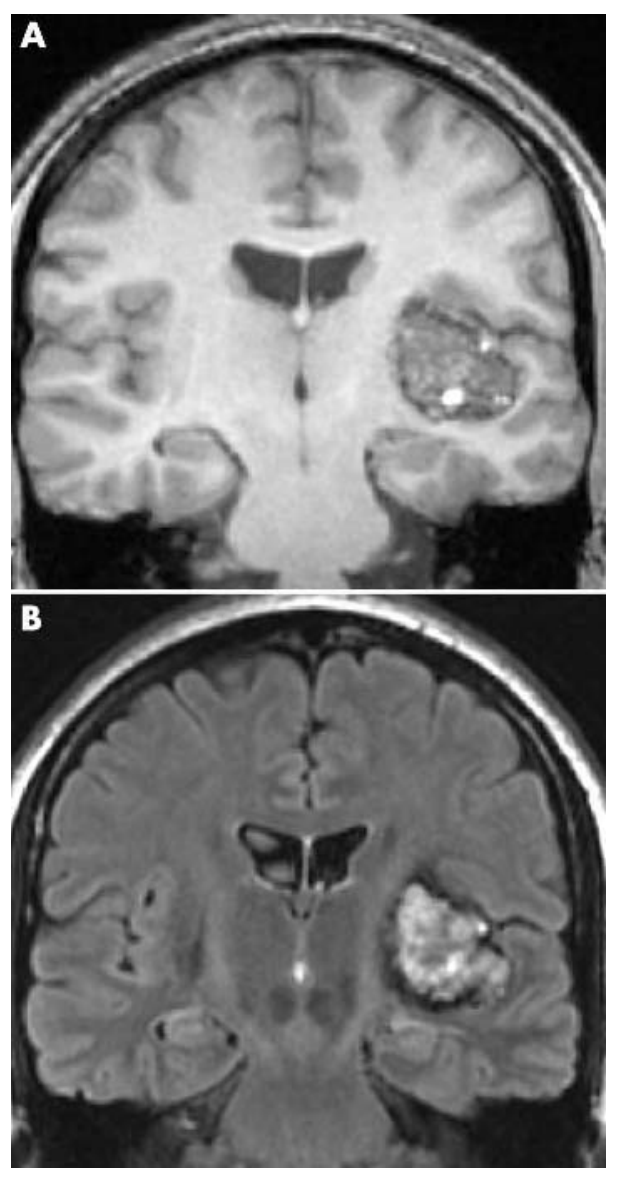

Figure 4 Cavernoma on the left (coronal 3.0 T MRI). (A) IRprep T1 weighted image. (B) T2 weighted FLAIR image. Both acquisitions demonstrate heterogeneous hyperintense signal caused by blood products in different stages of evolution, surrounded by a rim of low signal intensity from haemosiderin.

duration of epilepsy and a greater number of seizures. Longitudinal studies, however, are necessary to ascribe cause and effect. Changes in both hippocampal volume and T2 relaxation time have been found in serial MRI studies following prolonged early childhood convulsions. Neocortical, hippocampal, and cerebellar volume loss have been reported in follow up studies of adult patients with chronic epilepsy, implying that secondary brain damage may occur. The aims of future longitudinal studies are to identify those patients at risk of progressive damage and to develop surrogate markers of outcome for assessing the efficacy of disease modifying drugs.

\section{Novel MRI techniques}

The sensitivity of MRI can be enhanced by new data acquisition and processing techniques. Image processing such as analysis of the texture, curvilinear reformatting, and three dimensional reconstruction of the neocortex may help to identify abnormal gyral patterns or subtle FCDs. Voxel based morphometry provides an automated quantitative analysis of the distribution of grey and white matter and may be applied to individuals, and to groups of patients. The technique has demonstrated neocortical grey matter abnormalities in TLE patients with hippocampal atrophy and in patients with juvenile myoclonic epilepsy. Newly developed MRI contrasts magnetisation transfer imaging, fast FLAIR 

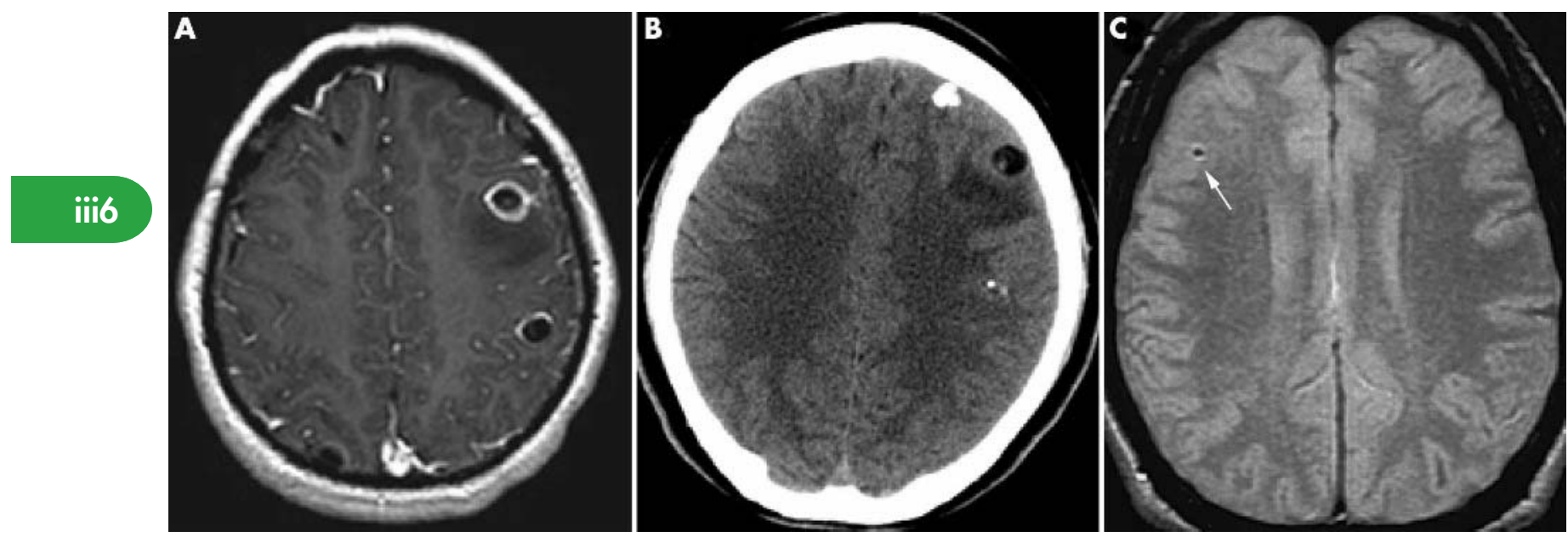

Figure 5 Cysticercosis. (A) Multiple ring enhancing lesions with surrounding oedema represent the inflammatory reactive stage of cysticercosis on this contrast enhanced T1 weighted axial image. (B) Corresponding CT scan shows calcification of these lesions. (C) In a different patient, the only MR abnormality was the punctuate signal void (arrow) in the right frontal lobe on this proton density weighted image. This was surgically proven to represent a calcified granuloma due to cysticercosis that caused chronic epilepsy. Figure courtesy of Professor Richard A Bronen, Yale University School of Medicine, USA. Original source: Bronen RA, Fulbright RK, Kim JH, et al. A systematic approach for interpreting MR images of the seizure patient. AJR Am J Roentgenol 1997;169:241-7; reproduced with permission.

T2, double inversion recovery and diffusion tensor imaging analysed with the voxel based approach are sensitive for detecting both developmental and acquired lesions in epilepsy, and identify focal abnormalities in some patients with refractory focal epilepsy and unremarkable conventional MRI scans. When used in combination these techniques may enable successful presurgical assessment for a greater number of patients with intractable epilepsy.

Diffusion weighted imaging (DWI) can detect areas of reduced diffusivity co-localising with the electroclinical seizure focus in patients with focal status epilepticus. Postictal imaging has also demonstrated decreases in diffusion after single short seizures. The observed changes are thought to reflect cellular swelling in the areas of seizure onset and spread. Diffusion tensor imaging (DTI) is a development of DWI, which allows better delineation of the magnitude of diffusion as well as identifying the principal direction of diffusion in any voxel. DTI maps can be used to identify nerve fibre tracts through the brain and to demonstrate the structural basis of connectivity between brain regions. In patients being considered for surgery, tractography can be used to map the optic radiation and the connections of eloquent areas in vivo, in order to minimise the risk of causing a deficit. Continuous arterial spin labelling perfusion MRI assesses cerebral blood flow and can detect asymmetries in interictal mesial temporal lobe perfusion in patients with TLE. The technique is potentially a further useful non-invasive tool for investigation of both interictal and ictal states. If a patient is restless and can only tolerate short studies the new ultra fast, low angle rapid acquisition and relaxation enhancement may be a useful sequence.

Improved gradient performance is anticipated to improve speed and spatial resolution. Phased array surface coils improve signal-to-noise ratio in superficial cortex and hippocampal regions and this also leads to improved spatial resolution. Imaging at high field strengths may also improve spatial resolution and 3T MRI scanners are now available as clinical instruments.

\section{Functional magnetic resonance imaging}

Functional MRI (fMRI) offers a tool for visualising regional brain activity. The areas detected with changes in blood oxygenation level dependent (BOLD) contrast reflect local changes in the ratio of deoxy- and oxyhemoglobin that occur during cognitive, sensory, and other tasks and allow the mapping of neural networks involved in the performance of these tasks. The most important clinical application of fMRI is in the localisation and lateralisation of cognitive functions of epilepsy patients evaluated for surgery in order to minimise the risk of causing a fixed deficit. Non-invasive fMRI has excellent spatial resolution and can be repeated as often as indicated. However, the studies require patient cooperation, both in performing tasks and limiting motion. For preoperative fMRI, it is important to choose activation procedures that are appropriate tests of function in the area of brain to be resected.

\section{Language}

Functional MRI provides a reliable way to lateralise language dominance. There may be considerable plasticity of language representation in the brains of epilepsy patients. Functional MRI has indicated that a high proportion (33\%) of intractable left TLE patients have bilateral or right hemispheric language related lateralisation. When used for the purpose of lateralisation of language, fMRI eliminates the need for invasive intracarotid amobarbital test (IAT) in $80 \%$ or more of patients. Overt disagreement between the IAT and fMRI is uncommon, although some discrepancy should be expected because of fundamental differences in the nature of the methods. A series of related tests, such as verbal fluency and language comprehension, should be performed for functional language mapping. Functional MRI reading tasks can identify language dominance in frontal and temporal areas. While current paradigms may indicate cerebral areas involved in receptive and expressive language functions, these data cannot at present be used to reliably guide surgical excision to minimise risk to language function. It cannot be assumed that all activated areas are crucial for language 
functions, or that areas that do not activate are not functionally important.

\section{Memory}

In addition to language, the assessment of memory functions dependent on the hippocampus is critical for planning anterior temporal resection. Functional MRI imaging of memory encoding and retrieval paradigms demonstrate that a wide range of stimuli can cause activation in mesial temporal structures. In normal individuals a verbal memory encoding task involves activation of the left hippocampus, whereas in patients with left hippocampal sclerosis the task is associated with reorganisation of activation to right hippocampus and parahippocampal gyrus. It is likely that fMRI with memory paradigms will be incorporated into the presurgical assessment in the coming years to minimise the adverse cognitive sequelae of anterior temporal lobe resection, and will result in less use of the IAT.

\section{Sensory and motor}

Sensory and motor tasks are the most reliable and reproducible of fMRI paradigms. They may be used to identify sensorimotor cortex when planning frontal or parietal neocortical resections.

\section{Seizure foci}

Ictal fMRI recordings are very difficult to obtain and are usually confounded by movement, but fMRI can be used to localise interictal epileptic activity. Technical developments enable simultaneous acquisition of EEG and fMRI data, and recent EEG-fMRI studies have shown reproducible BOLD activations concordant with EEG focus. Interictally spike related activation has been demonstrated in the perisylvian central region in benign childhood epilepsy with centrotemporal spikes. Further, bilateral activation of thalami and widespread cortical deactivation with a frontal maximum can be found in patients with idiopathic generalised epilepsy. Reflex epilepsies with induced seizure activity provide opportunities to image generators of interictal activity and the propagation of ictal activity. EEG-fMRI may aid EEG interpretation and understanding of the pathophysiological basis of epileptic activity. However, the approach must be evaluated and validated before being used clinically.

\section{Magnetic resonance spectroscopy}

Magnetic resonance spectroscopy (MRS) provides measurements of specific brain metabolites. Spectra are most commonly acquired from nuclei ${ }^{1} \mathrm{H}$ and ${ }^{31} \mathrm{P}$ using either single voxels or chemical shift imaging. Metabolites that are detectable with ${ }^{1} \mathrm{H}$ MRS include $\mathrm{N}$-acetylaspartate (NAA), choline, creatine, lactate, $\gamma$-aminobutyric acid (GABA), and glutamate. Compared to ${ }^{31} \mathrm{P}$ MRS measuring phosphorus containing compounds, ${ }^{1} \mathrm{H}$ MRS has greater signal-to-noise ratio and better spatial resolution, and it is also more easily carried out with MRI in a single examination. There is evidence that NAA is located primarily within neurons and precursor cells. Creatine and choline are found in both neurons and glia. Accurate definition of brain anatomy and the identification of structural abnormalities with MRI are necessary for the interpretation of MRS.

\section{Temporal lobe epilepsy}

${ }^{1} \mathrm{H}$ MRS lateralises the seizure focus in up to $80-90 \%$ of the TLE patients. Patients with hippocampal sclerosis show a decrease in NAA and increases in choline, creatine, and myo-inositol signals ipsilaterally. Low hippocampal NAA together with elevated glutamate + glutamine values have also been reported in patients with normal MRI. Twenty to fifty five per cent of patients with unilateral TLE have bilateral temporal abnormalities in ${ }^{1} \mathrm{H}$ MRS. Low NAA values have also been found in extratemporal neocortex in TLE. These widespread changes suggest that MRS abnormalities reflect cellular dysfunction-not merely neuronal loss or gliosis.

The role of ${ }^{1} \mathrm{H}$ MRS in predicting outcome of temporal lobe epilepsy surgery is not clear. Bilateral metabolic changes have been associated with poor outcome in TLE, especially if the preoperative contralateral abnormality is greater than the ipsilateral one. Metabolic abnormalities detected in ${ }^{1} \mathrm{H}$ MRS may be transient and normalisation of the contralateral NAA can occur postoperatively in seizure-free patients.

\section{Extratemporal epilepsy}

Frontal lobe epilepsy is associated with altered metabolic spectra with reduced $\mathrm{NAA} /$ creatine or $\mathrm{NAA} /$ choline ratios ipsilateral to the seizure focus. Thus ${ }^{1} \mathrm{H}$ MRS may help to lateralise a frontal focus in MRI negative patients. Bilateral abnormalities may be detected in FLE patients with unilateral focal EEG findings, and the clinical significance of this is less clear. It has been demonstrated that different types of MCDs show different degrees of decrease of NAA. ${ }^{1} \mathrm{H}$ MRS may also reveal metabolic abnormalities in the areas extending at a distance from the lesion and not evident on MRI. It is not certain whether this reflects more widespread pathology that is not evident on MRI, or is a functional consequence to an epileptic focus. Decreasing NAA has been correlated with increasing seizure frequency in FLE, suggesting that seizures are associated with neuronal dysfunction or loss.

\section{Generalised epilepsies}

${ }^{1} \mathrm{H}$ MRS studies in patients with idiopathic generalised epilepsy have provided evidence of thalamic dysfunction. A negative correlation has been found between NAA/creatine and the duration of epilepsy, and a relation between frequent generalised tonic-clonic seizures and low thalamic NAA concentrations. ${ }^{1} \mathrm{H}$ MRS also shows reduced frontal lobe concentrations of NAA in patients with juvenile myoclonic epilepsy.

\section{Postictal MRS}

Increased lactate and inorganic phosphate and decreased intracellular $\mathrm{pH}$ and phosphomonoesters have been detected peri- and postictally at the side of seizure focus. Elevation of lactate may last for a few hours after complex partial seizures and could therefore constitute a marker for the ictal zone. Lactate and choline increase and NAA decreases focally during prolonged non-convulsive seizures.

\section{MRS studies of neurotransmitters}

Low concentrations of GABA, the principal inhibitory neurotransmitter of brain, and homocarnosine have been detected using ${ }^{1} \mathrm{H}$ MRS in the occipital lobes of patients with frequent complex partial seizures. Higher concentrations of homocarnosine are associated with better seizure control in both patients with complex partial seizures and with juvenile myoclonic epilepsy. Numerous studies have reported an elevation of brain GABA concentrations after administration of vigabatrin, gabapentin, and topiramate. 
Changes in glutamate, the main excitatory neurotransmitter of the cortex, play an important role in the control of cortical excitability. Hippocampal specimens obtained at temporal lobectomy show increased intracellular glutamate content using ${ }^{1} \mathrm{H}$ MRS. Increases in glutamate and glutamine concentrations have also been measured after focal motor status epilepticus.

In summary, ${ }^{1} \mathrm{H}$ MRS may assist for lateralisation of the epileptic focus in TLE. The zone of altered metabolism extends beyond the structural and electrographic abnormalities, and may allow detection of pathologies in patients with normal MRI. Measurements of cerebral neurotransmitters, particularly GABA, glutamate, and glutamine, provide important information on the mechanisms related to epilepsy, as well as modes of action of antiepileptic drugs. Rapid developments in MR technology will improve spectral resolution and facilitate the investigation of metabolic dysfunction in epilepsy.

\section{Single photon emission computed tomography}

Single photon emission computed tomography (SPECT) is a nuclear medicine imaging method that allows measurements of regional cerebral blood flow changes in the areas affected by epileptic activity. Radioligands principally used in SPECT studies are ${ }^{99 \mathrm{~m}}$ Tc-hexamethyl-propylenamine oxime $\left({ }^{99 \mathrm{~m}} \mathrm{Tc}-\right.$ HMPAO) and technetium- ${ }^{99 \mathrm{~m}}$ Tc-cysteinate dimer (ECD, bicisate). Currently available stabilised forms of ${ }^{99 \mathrm{~m}}$ TcHMPAO and ECD are stable in vitro for several hours, whereas unstabilised ${ }^{99 \mathrm{~m}}$ Tc-HMPAO needs to be reconstructed immediately before intravenous injection. Seventy five per cent of ${ }^{99 \mathrm{~m}} \mathrm{Tc}$-HMPAO is extracted across the bloodbrain barrier, reaching peak concentrations within one minute after injection. The images can then be acquired up to six hours after tracer injection. Both ictal and postictal SPECT studies should be performed during simultaneous video-EEG monitoring to determine the relation between seizure onset and tracer injection. Interictal SPECT images serve as a reference baseline study for the interpretation of ictal images.

\section{Temporal lobe epilepsy}

Ictal

Ictal ${ }^{99 \mathrm{~m}}$ Tc-HMPAO SPECT is highly sensitive and specific in localising seizure onset in intractable TLE. Correct localisation of complex partial seizures may be achieved in over $90 \%$ of TLE patients. The use of subtraction ictal SPECT COregistered to MRI (SISCOM) improves the rate of localisation, and is particularly useful in patients with MCDs, or if there is no apparent lesion in the MRI. A characteristic pattern observed in temporal lobe seizures is an initial hyperperfusion of the temporal lobe, followed by medial temporal hyperperfusion and lateral temporal hypoperfusion. Analysis of ictal SPECT data with statistical parametric mapping (SPM) has shown more widespread activations in the cortico-thalamo-hippocampal-insular network of TLE. Hypoperfusion observed in the anterior frontal cortex may reflect ictal inhibition or deactivation.

Postictal SPECT injections are easier to perform than ictal injections, but the images are more difficult to interpret and have lower sensitivity and specificity. Temporal lobe seizures are correctly localised in $70 \%$ of the patients with postictal SPECT. The problem of seizure propagation and nonlocalisation caused by an early switch from ictal hyperperfusion to postictal hypoperfusion may be helped by setting up self injection or automated injection systems to facilitate early ictal injections.

Interictal

TLE patients show circumscribed hypoperfusion in interictal SPECT performed with ${ }^{99 \mathrm{~m}} \mathrm{Tc}-\mathrm{HMPAO}$ or with other cerebral blood flow ligands. The method is only moderately sensitive in localising a temporal lobe seizure focus $(40-50 \%$ correct localisation), has a relatively high false positive rate, and in consequence is not clinically useful as a stand alone test.

\section{Extratemporal epilepsy}

Ictal

Extratemporal seizures are often brief and it is therefore difficult to obtain an ictal recording. If true ictal ${ }^{99 \mathrm{~m}} \mathrm{Tc}$ HMPAO SPECT is obtained, accurate localisation of an extratemporal seizure focus may be possible in $90 \%$ of the cases using the SISCOM technique. Ictal SPECT demonstrates ipsilateral frontal hyperperfusion in FLE. Activations may also be detected in the ipsilateral basal ganglia and contralateral cerebellum. The method provides additional information in patients with unrevealing EEG and MRI results, and can also be used to study the blood flow changes underlying specific clinical features observed in extratemporal seizures.

Postictal injections in extratemporal epilepsy are of limited localising value. Compared to the ictal studies the yield of postictal SPECT studies is much lower (correct localisation in $46 \%$ ), and usually only very early postictal studies are diagnostic. Interictal SPECT is an insensitive method for localising extratemporal foci.

In conclusion, ictal SPECT provides a complementary method for localisation of the seizure focus in patients with intractable focal epilepsy evaluated for surgical treatment. The investigation may be particularly valuable in patients with normal MRI and presumed extratemporal seizures in order to generate a hypothesis that may then be tested with intracranial EEG recordings.

Positron emission tomography

${ }^{18} \mathrm{~F}$-deoxyglucose (FDG) and ${ }^{15} \mathrm{O}$-water $\left(\mathrm{H}_{2}{ }^{15} \mathrm{O}\right)$

Positron emission tomography (PET) maps cerebral glucose metabolism using ${ }^{18} \mathrm{~F}$-deoxyglucose $\left({ }^{18} \mathrm{FDG}\right)$ and cerebral blood flow using ${ }^{15} \mathrm{O}$-labelled water. Interictally PET shows areas of reduced glucose metabolism and blood flow that usually include the seizure focus but are more extensive. Regional hypometabolism is best analysed with co-registration of PET scans to MR images. Voxel based SPM has been shown to be useful in clinical evaluation of the data, and quantitative analysis with correction for partial volume effects further improves the accuracy of the method. The spatial resolution of quantitative ${ }^{18}$ FDG-PET is superior to SPECT or MRS. Ictal ${ }^{18}$ FDG-PET scans are difficult to obtain because cerebral uptake of ${ }^{18} \mathrm{FDG}$ occurs over 40 minutes after injection. Ictal ${ }^{15} \mathrm{O}-\mathrm{H}_{2} \mathrm{O}$ is problematic, because of the short half life of ${ }^{15} \mathrm{O}$ and unpredictable timing of seizure onsets.

Temporal lobe epilepsy

${ }^{18}$ FDG-PET detects interictal glucose hypometabolism ipsilateral to the seizure focus in $60-90 \%$ of TLE patients. Unilateral or asymmetric bilateral diffuse regional hypometabolism usually extends mesiolaterally in the temporal lobe. Some patients also have changes in extratemporal 
cortical areas or in the basal ganglia or thalamus. ${ }^{18}$ FDG-PET has some additional sensitivity over optimal, volumetric MRI but does not provide clinically useful information if hippocampal atrophy is present. ${ }^{15} \mathrm{O}-\mathrm{H}_{2} \mathrm{O}$ studies generally show hypoperfusion in the same areas as glucose hypometabolism, but are less sensitive and associated with more frequent false lateralisation.

${ }^{18}$ FDG-PET is more useful for lateralising than localising the epileptic focus. The sensitivity of the method differs according to the nature of underlying lesion, if any. Patients with hippocampal sclerosis have low glucose metabolism in the whole temporal lobe, while patients with mesiobasal temporal tumours show only a slight decrease in metabolism. Similar or elevated metabolic activity, compared to normal grey matter, has been detected in MCDs such as heterotopia. The metabolic pattern may be different in patients with mesial or lateral temporal seizure onset, but no clear correlation has been found between the degree of hypometabolism and the location of the epileptic focus.

Hippocampal sclerosis with neuronal cell loss and gliosis is the primary underlying cause of glucose hypometabolism in mesial TLE. Reduced glucose consumption has also been described in patients with mild or no hippocampal damage, suggesting that neurons are functioning with decreased synaptic activity. There have been few studies of newly diagnosed patients with TLE. In children with new onset temporal lobe seizures, hypometabolism is found in only $24 \%$ of the cases, whereas $80-85 \%$ of adults with intractable TLE have low glucose utilisation in the temporal lobe.

Unilateral focal temporal hypometabolism in ${ }^{18} \mathrm{FDG}$-PET predicts a good outcome of surgery for TLE. However, absence of reduced metabolism unilaterally does not preclude a favourable outcome. Symmetric, bilateral temporal hypometabolism is associated with higher incidence of postoperative seizures as are areas of severe extratemporal cortical, or thalamic hypometabolism. Postoperative increases have been found in inferior frontal and thalamic metabolism indicating that seizures may have a reversible effect on brain areas connected with the epileptic focus.

\section{Extratemporal epilepsy}

${ }^{18}$ FDG-PET has lower sensitivity for lateralisation of epileptic foci in extratemporal epilepsies than in TLE. The areas of decreased glucose metabolism are also less frequently well localised. Sixty per cent of patients with FLE show regional hypometabolism with ${ }^{18}$ FDG-PET, and a relevant underlying structural pathology is found on MRI in $90 \%$ of them. The area of hypometabolism may be either diffuse or widespread, or restricted to the co-localising MRI lesion. Statistical parametric mapping improves the diagnostic yield of ${ }^{18}$ FDG-PET, and assists the planning of implantation of intracranial electrodes in some intractable FLE patients with non-localising MRI or scalp EEG. In the majority of patients with FLE, however, ${ }^{18}$ FDG-PET does not appear to provide additional clinically useful information.

In summary, the place of interictal ${ }^{18}$ FDG-PET is in determining the lateralisation of epileptic focus, especially in the presurgical assessment of patients where there is not good concordance between MRI, EEG, and other data, in order to generate a hypothesis to be tested with intracranial EEG recordings. Developments in MRI have reduced the need of ${ }^{18}$ FDG-PET for localisation of an epileptic focus.
PET studies of specific ligands

PET may be used to demonstrate the binding of specific ligands-for example, ${ }^{11} \mathrm{C}$-flumazenil (FMZ) to the central benzodiazepine-GABA $\mathrm{A}_{\mathrm{A}}$ receptor complex (cBZR), ${ }^{11} \mathrm{C}$-diprenorphine and ${ }^{11} \mathrm{C}$-carfentanil to opiate receptors, and ${ }^{11} \mathrm{C}$ deprenyl to MAO-B. The technique is costly and scarce, but gives quantitative data with superior spatial resolution to SPECT.

\section{$G A B A_{A}$-benzodiazepine receptors: flumazenil}

${ }^{11} \mathrm{C}$-flumazenil (FMZ) is a useful marker of the $\mathrm{GABA}_{\mathrm{A}}-$ central benzodiazepine receptor (cBZR) complex.

\section{Temporal lobe epilepsy}

${ }^{11} \mathrm{C}$-FMZ-PET detects abnormalities in ${ }^{11} \mathrm{C}$-FMZ binding in over $90 \%$ of intractable TLE patients evaluated for surgery. The area of decreased ${ }^{11} \mathrm{C}-\mathrm{FMZ}$ binding is often smaller than that of glucose hypometabolism. In clinical terms, ${ }^{11} \mathrm{C}-\mathrm{FMZ}$ PET may be superior to ${ }^{18}$ FDG PET for the localisation of epileptic focus but does not provide additional useful information in the presurgical evaluation of patients with clear cut MRI findings of hippocampal sclerosis. ${ }^{11} \mathrm{C}-\mathrm{FMZ}$ PET also detects abnormalities in the medial temporal lobe of TLE patients with normal MRI. Potentially surgically useful reductions in hippocampal or extrahippocampal ${ }^{11} \mathrm{C}-\mathrm{FMZ}$ binding have been found in $47 \%$ of MRI negative TLE patients.

\section{Extratemporal epilepsy}

Studies of extratemporal epilepsy patients including those with normal MRI have indicated that surgically useful abnormalities of ${ }^{11} \mathrm{C}$-FMZ binding can be found in half of the cases. In addition to decreased ${ }^{11} \mathrm{C}-\mathrm{FMZ}$ binding, focal increases have been reported in MRI negative patients possibly indicating an occult developmental lesion. In patients with MCD ${ }^{11} \mathrm{C}$-FMZ abnormalities frequently extend beyond the visible MRI lesion which could explain why surgical treatment of MCD is less successful than treatment of discrete lesions.

\section{Other PET tracers}

The opioid system can be investigated with ${ }^{11} \mathrm{C}$-carfentanil, ${ }^{11} \mathrm{C}$-methylnaltrindole, and ${ }^{11} \mathrm{C}$-diprenorphine and the serotonin system with $\alpha$-[11C]methyl-L-tryptophan ([11C]AMT). These PET tracers provide interesting research data, but are not currently applied in clinical practice.

In summary, PET offers a tool for investigating neurochemical abnormalities associated with epilepsies. The method is an important research tool and can be useful in selected clinical situations, especially when there is not good concordance between MRI, EEG, and other data. Further ligands, particularly tracers for excitatory amino acid receptors, subtypes of the opioid receptors and the $G_{A B A}$ receptor, will improve the characterisation of different epileptic syndromes.

Authors' affiliations

T M Salmenpera, J S Duncan, The MRI Unit, The National Society for Epilepsy and the Department of Clinical and Experimental Epilepsy, Institute of Neurology, University College London, London, UK

Competing interest statement: The authors wish to declare no competing interests 


\section{REFERENCES}

1 International League Against Epilepsy. Recommendations for neuroimaging of patients with epilepsy. Commission on neuroimaging of the International League Against Epilepsy. Epilepsia 1997;38:1255-6.

- Systematic general guidelines for the structural imaging of patients with newly diagnosed epilepsy and those with chronic epilepsy who have not been fully evaluated

2 International League Against Epilepsy. Guidelines for neuroimaging evaluation of patients with uncontrolled epilepsy considered for surgery. Commission on neuroimaging of the International League Against Epilepsy. Epilepsia 1998;39:1375-6.

- Structural imaging guidelines for patients with intractable epilepsy who are being evaluated for surgery. A useful supplement to the "Recommendations for neuroimaging of patients with epilepsy"

3 International League Against Epilepsy. Commission on diagnostic strategies. Recommendations for functional neuroimaging of persons with epilepsy. Neuroimaging subcommission of the International League Against Epilepsy. Epilepsia 2000;41:1350-6.

- Evaluation of the roles of traditional functional imaging techniques and the emerging MR based functional imaging methods both in clinical practice and research

4 Detre JA. FMRI: applications in epilepsy. Epilepsia 2004;45:26-31.

- A review of the physiological basis for functional MRI and its applications to epilepsy.

5 Duncan JS. Imaging and epilepsy. Brain 1997;120:339-77.

- A comprehensive review of the structural and functional imaging methods in the investigation and management of epilepsy.

6 Hammen T, Stefan H, Eberhardt KE, et al. Clinical applications of $1 \mathrm{H}-\mathrm{MR}$ spectroscopy in the evaluation of epilepsies - what do pathological spectra stand for with regard to current results and what answers do they give to common clinical questions concerning the treatment of epilepsies? Acta Neurol Scand 2003; 108:223-38

- An overview of the principles and clinical applications of IH-MRS with special regard to the temporal lobe epilepsies.

7 Hammers A. Flumazenil positron emission tomography and other ligands for functional imaging. Neuroimag Clin N Am 2004;14:537-51.

- This review focuses on the use of flumazenil PET and other ligands in focal and idiopathic generalised epilepsies.

8 Henry TR, Van Heertum RL. Positron emission tomography and single photon emission computed tomography in epilepsy care. Semin Nud Med 2003;33:88-104.

- Emission tomographic imaging methodology, findings in clinical epilepsy studies and in the presurgical evaluation are discussed in detail in this overview.

9 Koepp MJ, Duncan JS. Epilepsy. Curr Opin Neurol 2003;17:467-74.

- An interesting review of the recent findings and potential role of neuroimaging from an epilepsy perspective.

10 Rugg-Gunn FJ, Symms MR. Novel MR contrasts to reveal more about the brain. Neuroimag Clin N Am 2004;14:449-70.

- This article describes novel MR contrasts which have the potential to identify some of the discrete focal abnormalities not detected on current conventional optimal MR imaging.

11 Vattipally VR, Bronen RA. MR imaging of epilepsy: strategies for successful interpretation. Neuroimag Clin N Am 2004;14:373-400.

- A useful introduction and overview for MR imaging of epilepsy. This review also discusses strategies for successful interpretation of MR images from the seizure patient and differential diagnosis to avoid potential piffalls. 